\title{
WELL-SEPARATED PAIR DECOMPOSITION FOR THE UNIT-DISK GRAPH METRIC AND ITS APPLICATIONS*
}

\author{
JIE $\mathrm{GAO}^{\dagger}$ AND LI ZHANG ${ }^{\ddagger}$
}

\begin{abstract}
We extend the classic notion of well-separated pair decomposition [P. B. Callahan and S. R. Kosaraju, J. ACM, 42 (1975), pp. 67-90] to the unit-disk graph metric: the shortest path distance metric induced by the intersection graph of unit disks. We show that for the unit-disk graph metric of $n$ points in the plane and for any constant $c \geq 1$, there exists a $c$-well-separated pair decomposition with $O(n \log n)$ pairs, and the decomposition can be computed in $O(n \log n)$ time. We also show that for the unit-ball graph metric in $k$ dimensions where $k \geq 3$, there exists a $c$-wellseparated pair decomposition with $O\left(n^{2-2 / k}\right)$ pairs, and the bound is tight in the worst case. We present the application of the well-separated pair decomposition in obtaining efficient algorithms for approximating the diameter, closest pair, nearest neighbor, center, median, and stretch factor, all under the unit-disk graph metric.
\end{abstract}

Key words. well-separated pair decomposition, unit-disk graph, approximation algorithm

AMS subject classifications. 68W25, 68W40, 68R10, 05C 85

DOI. $10.1137 /$ S0097539703436357

1. Introduction. Well-separated pair decomposition, introduced by Callahan and Kosaraju [10], has found numerous applications in solving proximity problems for points in the Euclidean space $[8,10,9,5,4,29,25,19,14]$. A pair of point sets $(A, B)$ is $c$-well-separated if the distance between $A, B$ is at least $c$ times the diameters of both $A$ and $B$. A well-separated pair decomposition of a point set consists of a set of well-separated pairs that cover all the pairs of distinct points, i.e., any two distinct points belong to the different sets of some pair. In [10], Callahan and Kosaraju showed that for any point set in an Euclidean space and for any constant $c \geq 1$, there always exists a $c$-well-separated pair decomposition with linearly many pairs. This fact has been very useful in obtaining nearly linear time algorithms for many problems, such as computing $k$-nearest neighbors, $N$-body potential fields, geometric spanners, and approximate minimum spanning trees. Well-separated pair decomposition is also shown to be very useful in obtaining efficient dynamic, parallel, and external memory algorithms $[8,9,10,7,18]$.

The definition of well-separated pair decomposition can be naturally extended to any metric space. Curiously enough, however, there has been no work for such an extension. A possible reason is that a general metric space may not admit a well-separated pair decomposition with a subquadratic size. Indeed, even for the metric induced by a star tree with unit weight on each edge, ${ }^{1}$ any well-separated pair decomposition requires quadratically many pairs. This makes the well-separated pair decomposition useless for such a metric. In this paper, we will show that for a certain metric, there do exist well-separated pair decompositions with almost linear size, and

* Received by the editors October 16, 2003; accepted for publication (in revised form) February 2, 2005; published electronically October 3, 2005.

http://www.siam.org/journals/sicomp/35-1/43635.html

${ }^{\dagger}$ Center for the Mathematics of Information, California Institute of Technology, Pasadena, CA 91125 (jgao@ist.caltech.edu). This work was done while the author was at the Department of Computer Science, Stanford University, Stanford, CA 94305.

${ }^{\ddagger}$ Hewlett-Packard Labs, 1501 Page Mill Road, Palo Alto, CA 94304 (1.zhang@hp.com).

${ }^{1} \mathrm{~A}$ metric induced by a graph (with positive edge weights) is the shortest path distance metric of the graph. 
therefore many proximity problems under that metric can be solved efficiently. The metric we investigate is the so-called unit-disk graph metric. At the same time we call the well-separated pair decomposition in the Euclidean space the geometric wellseparated pair decomposition, to be distinguished from the decomposition in graph metrics.

For a point set $S$ in the plane, its unit-disk graph [12] is formed by connecting two points $p, q$ in $S$ if the Euclidean distance d $(p, q)$ is at most 1. A unit-disk graph can also be viewed as the intersection graph of a set of unit disks centered at the points in $S$. We consider the weighted unit-disk graphs where each edge $(p, q)$ receives the weight $\mathrm{d}(p, q)$. Similarly, unit-ball graphs can be defined for points in high dimensions. Such graphs have been used extensively to model the communication or influence between objects $[27,21]$ and studied in many different contexts $[12,6,22,15]$. For example, wireless ad hoc networks can be modeled by unit-disk graphs [23,30,31], as two wireless nodes can directly communicate with each other only if they are within a certain distance. In unsupervised learning, for a dense sampling of points from some unknown manifold, the length of the shortest path on the unit-ball graph is a good approximation of the geodesic distance on the underlying (unknown) manifold if the radius is chosen appropriately $[34,16]$. In this paper, we show that the allpairs shortest path lengths of nodes in unit-disk graphs (or unit-ball graphs) can be compactly encoded and efficiently estimated by a subquadratic size well-separated pair decomposition.

2. Overview. In this paper, we show that for the metric induced by the unitdisk graph on $n$ points and for any constant $c \geq 1$, there does exist a $c$-well-separated pair decomposition with $O(n \log n)$ pairs, and such a decomposition can be computed in $O(n \log n)$ time. We also show that the bounds can be extended to higher dimensions: for $k \geq 3$, there always exists a $c$-well-separated pair decomposition with size $O\left(n^{2-2 / k}\right)$ for the unit-ball graph metric on $n$ points, and the bound is tight in the worst case. The construction time is $O\left(n^{4 / 3} \operatorname{polylog} n\right)$ for $k=3$ and $O\left(n^{2-2 / k}\right)$ for $k \geq 4$.

The difficulty in obtaining a well-separated pair decomposition for unit-disk graph metric is that two points that are close in the space are not necessarily close under the graph metric. We first prove the bound for the point set with constant-bounded density, i.e., a point set where any unit disk covers only a constant number of points in the set, by using a packing argument similar to the one in [20]. For a point set with unbounded density, we apply the clustering technique similar to the one used in [17] to the point set and obtain a set of clusterheads with a bounded density. We then apply the result for bounded density point set on those clusterheads. Then, by combining the well-separated pair decomposition for the bounded density point sets and for the Euclidean metric, we are able to show that the bound holds for any point sets.

For a pair of well-separated sets, the distance between two points from different sets can be approximated by the distance between the two sets or the distance between any pair of points in different sets. In other words, a well-separated pair decomposition can be thought of as a compressed representation to approximate the $\Theta\left(n^{2}\right)$ pairwise distances. Many problems that require checking of the pairwise distances can therefore be approximately solved by examining those distances between the well-separated pairs of sets. When the size of the well-separated pair decomposition is subquadratic, it often gives us more efficient algorithms than examining all the pairwise distances. Indeed, this is the intuition behind many applications of the geometric well-separated 
pair decomposition. By using the same intuition, we show the application of wellseparated pair decomposition in several proximity problems under the unit-disk graph metric.

Specifically, we consider the following natural proximity problems. Assume that $S_{1} \subseteq S$.

- Furthest neighbor, diameter, center. The furthest neighbor of $p \in S_{1}$ is the point in $S_{1}$ that maximizes the distance to $p$. Related problems include computing the diameter, the maximum pairwise shortest distance for points in $S_{1}$, and the center, the point that minimizes the maximum distance to all the other points.

- Nearest neighbor, closest pair. The nearest neighbor of $p \in S_{1}$ is the point in $S_{1}$ with the minimum distance to $p$. Related problems include computing the closest pair, the pair with minimum shortest distance, and the bichromatic closest pair, the pair that minimizes distance between points from two different sets.

- Median. The median of $S$ is the point in $S$ that minimizes the average (or total) distance to all the other points.

- Stretch factor. For a graph $G$ defined on $S$, its stretch factor with respect to the unit-disk graph metric is defined to be the maximum ratio $\pi_{G}(p, q) / \pi(p, q)$, where $\pi_{G}, \pi$ are the distances induced by $G$ and by the unitdisk graph, respectively.

All the above problems can be solved or approximated efficiently for points in the Euclidean space. However, for the metric induced by a graph, even for planar graphs, very little is known other than solving the expensive all-pairs shortest path problem. For computing diameter, there is a simple linear time method that achieves a 2-approximation ${ }^{2}$ and a $4 / 3$-approximate algorithm with running time $O\left(m \sqrt{n \log n}+n^{2} \log n\right)$ for a graph with $n$ vertices and $m$ edges [2]. By using the powerful tool of the well-separated pair decomposition, we are able to obtain, for all the above problems, nearly linear time algorithms for computing 2.42 -approximation ${ }^{3}$ and $O\left(n \sqrt{n \log n} / \varepsilon^{3}\right)$ time algorithms for computing $(1+\varepsilon)$-approximation for any $\varepsilon>0$. In addition, the well-separated pair decomposition can be used to obtain an $O\left(n \log n / \varepsilon^{4}\right)$ space distance oracle so that any $(1+\varepsilon)$ distance query in the unit-disk graph can be answered in $O(1)$ time.

While the existence of almost linear size well-separated pair decomposition has reduced the number of pairs needed to examine when solving many proximity problems, we still need good approximation of the distances between those pairs. Our construction algorithm only produces well-separated pair decompositions without knowing an accurate approximation of the distances. For approximation algorithms, we need accurate estimation of shortest distances between $O(n \log n)$ pairs of points in the unitdisk graph. Indeed, the approximation ratio and the running time of our algorithms are dominated by the efficiency of such algorithms. Once the distance estimation has been made, the remaining computation only takes almost linear time.

For a general graph, it is unknown whether $O(n \log n)$-pairs shortest path dis-

\footnotetext{
${ }^{2}$ Select an arbitrary node $v$ and compute the shortest path tree rooted at $v$. Suppose that the furthest node from $v$ is of distance $D$ away. Then the diameter of the graph is no longer than $2 D$, by triangular inequality.

${ }^{3}$ For a minimization problem, a quantity $\hat{\ell}$ is a $c$-approximation of $\ell$ if $\ell \leq \hat{\ell} \leq c \ell$. An object $\hat{O}$ is a $c$-approximation of $O$ with respect to a cost function $f$ if $f(O) \leq f(\hat{O}) \leq c f(O)$. For a maximization problem, $\hat{\ell}$ is a $c$-approximation of $\ell$ if $\ell / c \leq \hat{\ell} \leq \ell$, and $\hat{O}$ is a $c$-approximation of $O$ if $f(O) / c \leq f(\hat{O}) \leq f(O)$.
} 
tances can be computed significantly faster than all-pairs shortest path distances. For the planar graph, one can compute $O(n \log n)$-pairs shortest path distance in $O(n \sqrt{n \log n})$ time by using separators with $O(\sqrt{n})$ size [3]. This method extends to the unit-disk graph with constant bounded density since such graphs enjoy similar separator property as the planar graphs [28, 32]. As for approximation, Thorup [35] recently discovered an algorithm for planar graphs that can answer any $(1+\varepsilon)$-shortest distance query in $O(1 / \varepsilon)$ time after almost linear time preprocessing. Unfortunately, Thorup's algorithm uses balanced shortest path separators in planar graphs which do not obviously extend to the unit-disk graphs. On the other hand, it is known that there does exist planar 2.42-spanner for a unit-disk graph [26]. By applying Thorup's algorithm to that planar spanner, we can compute 2.42-approximate shortest path distance for $O(n \log n)$ pairs in almost linear time.

Another application of well-separated pair decomposition is that we are able to obtain an almost linear size data structure to answer $(1+\varepsilon)$-approximate shortest path query in $O(1)$ time. Approximate distance oracles have been studied where the emphasis is often on the size of the oracles (for a survey, see [38]). For general graphs, it has been shown that it is possible to construct a $(2 k-1)$-approximate distance oracle with size $O\left(k n^{1+1 / k}\right)$ [36]. It is also shown in [36] that this bound is tight for some small $k$ 's and is conjectured to be tight for all the $k$ 's. For planar graphs, Thorup [35] and Klein [24] have shown that there exists $(1+\varepsilon)$-approximate distance oracle by using almost linear space for any $\varepsilon>0$. As mentioned, their results do not extend to the unit-disk graph. In addition, the query time of their algorithm is $O(1 / \varepsilon)$. Recently, Gudmundsson et al. showed that when a geometric graph is a Euclidean spanner, there does exist an almost linear time (and therefore almost linear space) method to construct $(1+\varepsilon)$-approximate and $O(1)$ query time distance oracles [19]. Again, a unit-disk graph is not necessarily a Euclidean spanner with bounded stretch factor, and their technique does not extend.

3. Definitions. Unit-disk graphs. Denote by $\mathrm{d}(\cdot, \cdot)$ the Euclidean metric. For a set of points $S$ in the plane, the unit-disk graph $I(S)=(S, E)$ is defined to be the weighted graph where an edge $e=(p, q)$ is in the graph if $\mathrm{d}(p, q) \leq 1$, and the weight of $e$ is $\mathrm{d}(p, q)$. Likewise, we can define the unit-ball graph for points in higher dimensions.

Metric space. Suppose that $(S, \pi)$ is a metric space where $S$ is a set of elements and $\pi$ the distance function defined on $S \times S$. For any subset $S_{1} \subseteq S$, the diameter $D_{\pi}\left(S_{1}\right)$ (or $D\left(S_{1}\right)$ when $\pi$ is clear from the context) of $S$ is defined to be $\max _{s_{1}, s_{2} \in S_{1}} \pi\left(s_{1}, s_{2}\right)$. The distance $\pi\left(S_{1}, S_{2}\right)$ between two sets $S_{1}, S_{2} \subseteq S$ is defined to be $\min _{s_{1} \in S_{1}, s_{2} \in S_{2}} \pi\left(s_{1}, s_{2}\right)$.

In this paper, we are interested in the unit-disk graph metric $\pi=\pi_{I(S)}$ induced by the unit-disk graph of a set of points $S$ in the plane, where the distance between any two nodes is defined to be the length of the shortest path between them.

Well-separated pair decomposition. For a metric space $(S, \pi)$, two nonempty subsets $S_{1}, S_{2} \subseteq S$ are called c-well-separated if $\pi\left(S_{1}, S_{2}\right) \geq c \cdot \max \left(D_{\pi}\left(S_{1}\right), D_{\pi}\left(S_{2}\right)\right)$.

Following the definition in [10], for any two sets $A$ and $B$, a set of pairs $\mathcal{P}=$ $\left\{P_{1}, P_{2}, \ldots, P_{m}\right\}$, where $P_{i}=\left(A_{i}, B_{i}\right)$, is called a pair decomposition of $(A, B)$ (or of $A$ if $A=B$ ) if

- for all the $i$ 's, $A_{i} \subseteq A$ and $B_{i} \subseteq B$;

- $A_{i} \cap B_{i}=\emptyset$;

- for any two elements $a \in A$ and $b \in B$, there exists a unique $i$ such that $a \in A_{i}$ and $b \in B_{i}$. We call $(a, b)$ is covered by the pair $\left(A_{i}, B_{i}\right)$. 


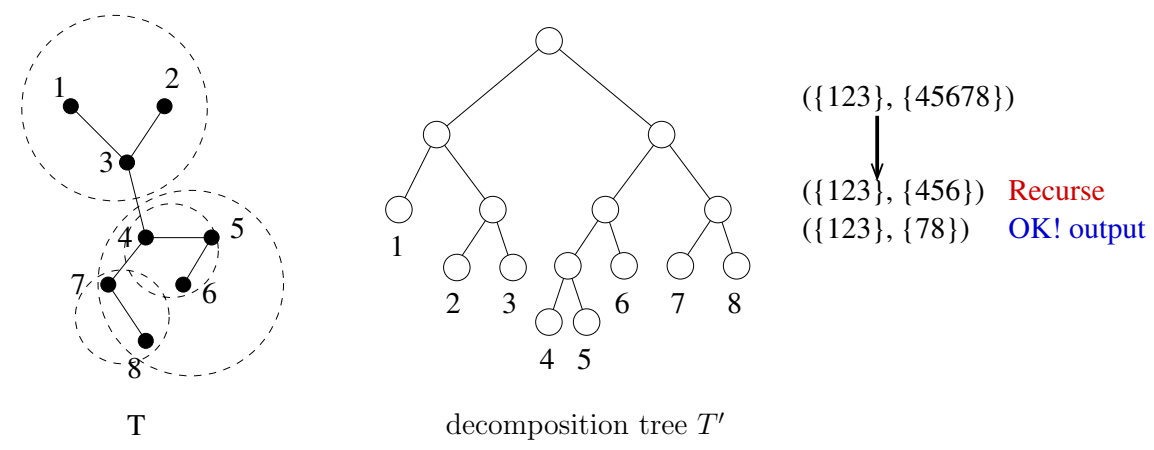

FIG. 4.1. An example of the decomposition tree.

If in addition, every pair in $\mathcal{P}$ is $c$-well-separated, $\mathcal{P}$ is called a $c$-well-separated pair decomposition ( $c$-WSPD). Clearly, any metric space admits a $c$-WSPD with quadratic size by using the trivial family that contains all the pairwise elements.

4. Well-separated pair decomposition for unit-disk graph metric. We start with the point set with constant bounded density. Then, by combining with geometric well-separated pair decomposition, we show the extension of the result to arbitrary point sets. We will focus our discussion on points in the plane, but most results extend to higher dimensions, resulting in subquadratic size well-separated pair decomposition. We also show that our bounds in $\mathbb{R}^{k}$ for $k \geq 3$ are tight.

4.1. Point sets with constant bounded density. The density $\alpha$ of a point set $S$ is defined to be the maximum number of points in $S$ covered by a unit disk. $S$ has constant bounded density if its density is $O(1)$. We assume that the unit-disk graph on $S$ is connected; otherwise, we can consider each connected component separately.

To construct a well-separated pair decomposition, we first compute the unit-disk graph $I(S)$ of $S$ and then a spanning tree $T$ of $I(S)$, where the maximum degree of $T$ is 6 . This can be done by computing the relative neighborhood graph of $S$ [37] and keeping those edges with length at most 1 . Let $G$ be the resulting graph. It is well known that $G$ is connected, and the degree of $G$ is at most 6 . We then compute a spanning tree of $G$. This step takes $O(n \log n)$ time [33]. It is also known that any $n$-vertex tree with maximum degree $\beta-1$ can be divided into two parts by removing a single edge so that each subtree contain at least $n / \beta$ vertices. We now recursively apply the balanced partitioning to obtain a balanced hierarchical decomposition of $T$ (see Figure 4.1). The decomposition can be represented as a rooted binary tree $T^{\prime}$ where each node $v \in T^{\prime}$ corresponds to a (connected) subtree $T(v)$ of $T$. The root of $T^{\prime}$ corresponds to $T$, and for a node $v \in T^{\prime}, v$ 's two children $v_{1}, v_{2}$ represent the two connected subtrees $T\left(v_{1}\right)$ and $T\left(v_{2}\right)$ obtained by removing an edge from $T(v)$. We denote by $S(v)$ the set of points in the subtree in $T(v)$. For a node $v \in T^{\prime}$, denote by $P(v)$ the parent node of $v$ in $T^{\prime}$. We also use $P(S(u))$ to denote $S(P(u))$. The height of the tree $T^{\prime}$ is obviously $O(\log n)$.

Now, we describe a procedure to produce a $c$-WSPD of $S$.

For each node $v \in T^{\prime}$, we pick an arbitrary point from $S(v)$ as a representative of $S(v)$ and denote it by $\sigma(S(v)$ ) (or $\sigma(v)$ ). We place in a queue the pair $(S(r), S(r)$ ), where $r$ is the root of $T^{\prime}$. We run the following process until the queue becomes empty: repeatedly remove the first element $\left(S\left(v_{1}\right), S\left(v_{2}\right)\right)$ from the queue. There are two cases: 
- $\mathrm{d}\left(\sigma\left(v_{1}\right), \sigma\left(v_{2}\right)\right) \geq(c+2) \cdot \max \left(\left|S\left(v_{1}\right)\right|-1,\left|S\left(v_{2}\right)\right|-1\right)$. In this case, we include the pair to $\mathcal{P}$.

- $\mathrm{d}\left(\sigma\left(v_{1}\right), \sigma\left(v_{2}\right)\right)<(c+2) \cdot \max \left(\left|S\left(v_{1}\right)\right|-1,\left|S\left(v_{2}\right)\right|-1\right)$. If $\left|S\left(v_{1}\right)\right|=\left|S\left(v_{2}\right)\right|=1$, then it must be the case that $S\left(v_{1}\right)$ and $S\left(v_{2}\right)$ contain the same point. In this case, we simply discard the pair. Otherwise, suppose that $\left|S\left(v_{1}\right)\right| \geq$ $\left|S\left(v_{2}\right)\right|$ and that $u_{1}, u_{2}$ are two children of $v_{1}$. We add to the queue two pairs $\left(S\left(u_{1}\right), S\left(v_{2}\right)\right)$ and $\left(S\left(u_{2}\right), S\left(v_{2}\right)\right)$.

The above process is very similar to the collision detection algorithm in [20] except that here a pair is produced when they are $c$-well-separated. We now make the following claims.

LEMma 4.1. $\mathcal{P}$ is a $c$-WSPD of $S$. Furthermore, each ordered pair of distinct points $(p, q)$ is covered by exactly one pair in $\mathcal{P}$.

Proof. By the construction, a pair $\left(S\left(v_{1}\right), S\left(v_{2}\right)\right)$ is included in $\mathcal{P}$ if and only if $\mathrm{d}\left(\sigma\left(v_{1}\right), \sigma\left(v_{2}\right)\right) \geq(c+2) \cdot \max \left(\left|S\left(v_{1}\right)\right|-1,\left|S\left(v_{2}\right)\right|-1\right)$. Since for any $v \in T^{\prime}, S(v)$ is connected, $D_{\pi}(S(v)) \leq|S(v)|-1$. In addition, $\pi(p, q) \geq \mathrm{d}(p, q)$. Thus, we have that

$$
\begin{aligned}
& \pi\left(S\left(v_{1}\right), S\left(v_{2}\right)\right) \\
\geq & \pi\left(\sigma\left(v_{1}\right), \sigma\left(v_{2}\right)\right)-\left(D_{\pi}\left(S\left(v_{1}\right)\right)+D_{\pi}\left(S\left(v_{2}\right)\right)\right. \\
\geq & \mathrm{d}\left(\sigma\left(v_{1}\right), \sigma\left(v_{2}\right)\right)-2 \max \left(\left|S\left(v_{1}\right)\right|-1,\left|S\left(v_{2}\right)\right|-1\right) \\
\geq & c \cdot \max \left(\left|S\left(v_{1}\right)\right|-1,\left|S\left(v_{2}\right)-1\right|\right) \\
\geq & c \cdot \max \left(D_{\pi}\left(S\left(v_{1}\right)\right), D_{\pi}\left(S\left(v_{2}\right)\right)\right) .
\end{aligned}
$$

That is, every pair in $\mathcal{P}$ is a $c$-well-separated pair. The process clearly ends. To argue that $\mathcal{P}$ covers all the pairs of distinct points, we observe that we begin with the pair $(S(r), S(r))$ that covers all the pairs, and each time when we split a node, the union of the pairs covered remain the same. The pairs we discard are of the form $(\{p\},\{p\})$. Thus, all the ordered pairs of distinct points are covered by $\mathcal{P}$. Since the splitting produces two disjoint sets, each ordered pair is covered exactly once.

The following lemma shows that the sizes of two sets in the same pair do not differ too much.

LEMma 4.2. Each pair $(A, B)$ that ever appears in the queue satisfies $1 / \beta \leq$ $|A| /|B| \leq \beta$.

Proof. The proof is done by induction. Clearly, it is true for the pair $(S(r), S(r))$. Now, consider the splitting that generates the pair $(A, B)$. Without loss of generality, assume that we split $P(B)$, the parent node of $B$. By the splitting rule, we have that $|A| \leq|P(B)|$. By induction hypothesis, $|A| \geq|P(B)| / \beta \geq|B| / \beta$. Since the splitting is balanced, $|B| \geq|P(B)| / \beta \geq|A| / \beta$. Therefore $1 / \beta \leq|A| /|B| \leq \beta$.

Now, we bound the size of $\mathcal{P}$.

Lemma 4.3. If $\left(A, B_{i}\right) \in \mathcal{P}, i=1, \ldots, m(A)$, then $B_{i} \cap B_{j}=\emptyset$, and $m(A)=$ $O\left(c^{2}|A|\right)$.

Proof. By Lemma 4.1, each pair of points can be covered only once; thus $B_{i} \cap B_{j}=$ $\emptyset$ if both $\left(A, B_{i}\right)$ and $\left(A, B_{j}\right)$ are in $\mathcal{P}$.

If $\left(A, B_{i}\right) \in \mathcal{P}$, then $\left(P(A), P\left(B_{i}\right)\right)$ is not in $\mathcal{P}$. So $\mathrm{d}\left(\sigma(P(A)), \sigma\left(P\left(B_{i}\right)\right)\right)<$ $(c+2) \cdot \max \left(|P(A)|-1,\left|P\left(B_{i}\right)\right|-1\right)$. Set $R=\beta|P(A)| \leq \beta^{2}|A|$. If we split $P\left(B_{i}\right)$ to get the pair $\left(A, B_{i}\right)$, then $\left(A, P\left(B_{i}\right)\right)$ appeared in the queue, by Lemma 4.2 , we have $\left|P\left(B_{i}\right)\right| \leq \beta|A| \leq \beta|P(A)|=R$. If we split $P(A)$ to get the pair $\left(A, B_{i}\right)$, then $\left|B_{i}\right| \leq|P(A)|$, so $\left|P\left(B_{i}\right)\right| \leq \beta\left|B_{i}\right| \leq \beta|P(A)|=R$. Then,

$$
\mathrm{d}\left(\sigma(P(A)), \sigma\left(P\left(B_{i}\right)\right)\right)<(c+2) R, D_{\pi}\left(P\left(B_{i}\right)\right) \leq R .
$$

Then all the points in $B_{i}$ must be inside a disk of radius $(c+3) R$ centered at $\sigma(P(A))$. Therefore we have that $\left|\cup_{i=1}^{m(A)} B_{i}\right|=O\left((c+3)^{2} R^{2}\right)$ because $S$ has constant 
bounded density. By Lemma 4.2, we know that $\left|B_{i}\right| \geq|A| / \beta \geq|P(A)| / \beta^{2}$. Thus, $\left|B_{i}\right| \geq R / \beta^{3}$. Then, we have that $m(A)=O\left((c+3)^{2} R^{2} /\left(R / \beta^{3}\right)\right)=O\left(c^{2} R\right)=$ $O\left(c^{2}|A|\right)$.

LEMma 4.4. $|\mathcal{P}|=O\left(c^{2} n \log n\right)$.

Proof. Define $V_{i}=\left\{v \in T^{\prime}|| S(v) \mid \in\left[2^{i}, 2^{i+1}\right)\right\}$ for $0 \leq i \leq \log n$. Clearly, $\left|V_{i}\right|=O\left(n / 2^{i}\right)$. Define $\Sigma_{i}=\left\{(S(v), B) \in \mathcal{P} \mid v \in V_{i}\right\}$. Denote by $m(S(v))$ the total number of pairs in which $S(v)$ is involved. By Lemma 4.3, we have that

$$
\begin{aligned}
\left|\Sigma_{i}\right| & =\sum_{v \in V_{i}} m(S(v))=\sum_{v \in V_{i}} O\left(c^{2}|S(v)|\right) \\
& =O\left(c^{2} 2^{i+1} \cdot n / 2^{i}\right)=O\left(c^{2} n\right) .
\end{aligned}
$$

Thus, $|\mathcal{P}|=\sum_{i=0}^{\log n}\left|\Sigma_{i}\right|=O\left(c^{2} n \log n\right)$.

Combining the above result, we now have the following theorem.

THEOREM 4.5. For any $n$ points with constant-bounded density in the plane and any $c \geq 1$, there exists a $c$-WSPD with $O\left(c^{2} n \log n\right)$ pairs, which can be computed in $O\left(c^{2} n \log n\right)$ time.

Proof. Clearly, the time needed is proportional to the number of pairs that ever appear in the queue. We can represent the construction as a tree: each pair corresponds to a node in the tree, and when a pair is split, we treat those two resulting pairs as the children of the pair. Clearly, the leaves of the tree correspond to those pairs included in $\mathcal{P}$ and the pairs discarded. All the discarded pairs have the form $(\{p\},\{p\})$, and there are $O(n)$ such pairs. Thus, the total number of nodes in the tree is bounded by $O(|\mathcal{P}|)=O\left(c^{2} n \log n\right)$. Each split costs $O(1)$. Therefore, the total computation cost is $O\left(c^{2} n \log n\right)$.

The result can be easily extended to the point set with maximum density $\alpha$.

COROLlary 4.6. For a point set with maximum density $\alpha$, for any $c \geq 1$, a $c-W S P D$ with $O\left(\alpha c^{2} n \log n\right)$ pairs can be constructed in $O\left(\alpha c^{2} n \log n\right)$ time.

Proof. If the point set has maximum density $\alpha$, Lemma 4.3 still holds if we change $m(A)$ to $O\left(\alpha c^{2}|A|\right)$. Substituting the value in Lemma 4.4, we have that $|\mathcal{P}|=O\left(\alpha c^{2} n \log n\right)$. The claim then follows from Theorem 4.5.

By a similar argument, we can extend the result to higher dimensions.

THEOREM 4.7. Given a point set in $\mathbb{R}^{k}$, where $k \geq 3$, with constant bounded density and any constant $c \geq 1$, there exist a $c$-WSPD with $O\left(n^{2-2 / k}\right)$ pairs for the unit-ball graph metric. This bound is tight in the worst case. And the decomposition can be computed in $O\left(n^{2-2 / k}\right)$ time.

Proof. We first compute a spanning tree of $S$ with constant maximum degree $\beta_{k}$, a constant dependent on $k$ only. This can be done by using the technique in [4]. We then follow the same process as described above. The upper bound follows from the same packing argument as in Lemma 4.4. Lemma 4.3 can be changed so that the number of pairs associated with a node $A$ is $m(A)=O\left(|A|^{k-1}\right)$. In addition, by Lemma 4.2, for any pair $(A, B) \in P, 1 / \beta_{k} \leq|A| /|B| \leq \beta_{k}$. Thus, $m(A)=O(n /|A|)$. Define $V_{i}$ as in Lemma 4.4, $\left|V_{i}\right|=O\left(n / 2^{i}\right)$. When $0 \leq i \leq \frac{1}{k} \log n,\left|\Sigma_{i}\right|=\sum_{v \in V_{i}} m(S(v))=$ $O\left(\sum_{v \in V_{i}}|S(v)|^{k-1}\right)=O\left(2^{i(k-1)} \cdot n / 2^{i}\right)=O\left(n 2^{i(k-2)}\right)$. When $i>\frac{1}{k} \log n,\left|\Sigma_{i}\right|=$ $\sum_{v \in V_{i}} m(S(v))=O\left(\sum_{v \in V_{i}} n /|S(v)|\right)=O\left(\left(n / 2^{i}\right)^{2}\right)$. Therefore,

$$
\begin{aligned}
|\mathcal{P}| & =\sum_{0 \leq i \leq \frac{1}{k} \log n} n 2^{i(k-2)}+\sum_{\frac{1}{k} \log n<i \leq \log n} O\left(n^{2} / 2^{2 i}\right) \\
& =O\left(n^{2-2 / k}\right) .
\end{aligned}
$$




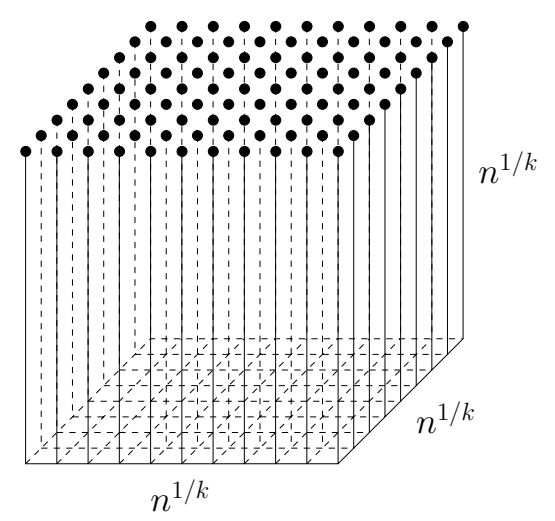

FIG. 4.2. A lower bound example of the well-separated pair decomposition for points in $k=3$ dimensions. The shaded dots are tip points.

As for the lower bound, consider the points on the $k$-dimensional grid $\left[0, n^{1 / k}\right) \times$ $\ldots \times\left[0, n^{1 / k}\right)$. Define a graph $G$ with edges between pairs of points $\left(x_{1}, \ldots, x_{i}, \ldots, x_{k}\right)$ and $\left(x_{1}, \ldots, x_{i}+1, \ldots, x_{k}\right)$ for $i=1$, or $x_{1}=0$ and $i \geq 2$. A point $\left(n^{1 / k}-1, x_{2}, \ldots, x_{k}\right)$ for $0 \leq x_{i}<n^{1 / k}$ is called a tip point. Intuitively, $G$ can be thought as a graph where the tip points dangle down from a $(k-1)$-dimensional mesh. See Figure 4.2. Clearly, we can perturb the point set so that its unit-ball graph equals $G$. The metric defined by $G$ has the following property: (i) the diameter of $G$ is $k n^{1 / k}$; (ii) the distance between any two tip points is at least $2 n^{1 / k}$. Therefore, when $c>k / 2$, a $c$-WSPD cannot have two tip points in the same set of a pair. Since there are $\Theta\left(n^{1-1 / k}\right)$ tip points, $\Omega\left(n^{2-2 / k}\right)$ pairs are needed, just to separate those tip points.

By the same argument as in Theorem 4.5, it is easily seen that the $c$-WSPD can be computed in $O\left(n^{2-2 / k}\right)$ time.

4.2. Arbitrary point sets. The packing argument fails for the unit-disk graph of point sets with unbounded density. However, we can reduce the problem to the constant density case by first clustering the points and then considering those crowded points separately by using geometric well-separated pair decompositions.

For $0 \leq \delta \leq 1$, a point $p$ is $\delta$-covered (or simply covered) by a point $s$ if $\mathrm{d}(s, p) \leq \delta$. Denote by $U(s)$ the set of points $\delta$-covered by $s$. A subset $X \subseteq S$ is called a $\delta$-cover of $S$ if any point in $S$ is $\delta$-covered by some point in $X$. We call the points in a $\delta$-cover $X$ clusterheads. For each point in $S$, we assign it to the nearest clusterhead. Thus $X$ induces a partitioning of $S$ into sets $C(s)=S \cap \operatorname{Vor}(s)$, where $\operatorname{Vor}(s)$ denotes the Voronoi cell of $s$ in $X$. Clearly, for any $p \in C(s), \mathrm{d}(s, p) \leq \delta$, i.e., $C(s) \subseteq U(s)$. A $\delta$-cover is called minimal if no two points in $X$ are within distance $\delta$ to each other. For any set $A \subseteq X$, denote by $\hat{A}$ the set $\hat{A}=\cup_{s \in A} C(s)$.

To deal with an arbitrary point set $S$, we first compute a minimal cover $X$ of $S$ with an appropriately chosen $\delta$. We then apply our results on constant-bounded density point sets to $X$. Note that we cannot use the unit-disk graph on $X$ because it may not have the same connectivity as the unit-disk graph on $S$. Denote any two points $s_{1}, s_{2}$ in $X$ neighbors if $\mathrm{d}\left(s_{1}, s_{2}\right)>1$, and there exist two points $p_{1} \in C\left(S_{1}\right)$ and $p_{2} \in C\left(s_{2}\right)$ such that $\mathrm{d}\left(p_{1}, p_{2}\right) \leq 1$. We call the pair $\left(p_{1}, p_{2}\right)$ a bridge between $s_{1}$ and $s_{2}$. For each neighboring pair, we only pick one bridge arbitrarily. Let $Y$ denote the set of all bridge points. Consider the point set $Z=X \cup Y$. Let $\pi^{\prime}$ denote the unit-disk graph metric on the set $Z$. Now, we make the claim in the next lemma. 
Lemma 4.8. $X$ has $O\left(1 / \delta^{2}\right)$-density. $Z$ can be computed in $O\left(n \log n / \delta^{2}\right)$ time.

Proof. Any two points $s_{1}, s_{2}$ in a minimal cover $X$ are of at least distance $\delta$ away from each other. Therefore, there are $O\left(1 / \delta^{2}\right)$ points of $X$ inside any unit disk. So $X$ has $O\left(1 / \delta^{2}\right)$ density.

To compute $X$, we can use a greedy algorithm with the assistance of a dynamic point location data structure of unit disks [11]. The algorithm runs in $O(n \log n)$ time. To compute all the neighboring pairs, we can enumerate all the pairs $\left(s_{1}, s_{2}\right)$, where $s_{2}$ is inside the square centered at $s_{1}$ and with side-length $2(1+2 \delta)$. There are $O\left(n / \delta^{2}\right)$ such pairs according to Lemma 4.8 , and they can be computed in $O\left(n \log n / \delta^{2}\right)$ time by using a standard rectangular range searching data structure. Call such pairs candidate pairs. Clearly, only a candidate pair can possibly be a neighboring pair. To find a bridge between two clusterheads $s_{1}, s_{2}$ of a candidate pair, we can compute the bichromatic closest pair between two sets $C\left(s_{1}\right), C\left(s_{2}\right)$. In the plane, this can be done in $O\left(\left|U\left(s_{1}\right) \cup U\left(s_{2}\right)\right| \log n\right)$ time. Since we need only to examine each clusterhead against $O\left(1 / \delta^{2}\right)$ clusterheads, the total computation time is bounded by $O\left(n \log n / \delta^{2}\right)[1]$.

Now we show that $\pi^{\prime}$ approximates $\pi$ well on the set $X$.

Lemma 4.9. For any two points $p, q \in X$,

$$
\pi(p, q) \leq \pi^{\prime}(p, q) \leq(1+12 \delta) \pi(p, q)+12 \delta .
$$

Proof. Since $Z \subseteq S, \pi(p, q) \leq \pi^{\prime}(p, q)$. On the other hand, assume that $p_{0} p_{1} \cdots p_{m}$, where $p_{0}=p$ and $p_{m}=q$ is a shortest path between $p$ and $q$ in the unit-disk graph of $S$. For $0 \leq i \leq m$, suppose that $s_{i}$ is the clusterhead that covers $p_{i}$. Note that $s_{0}=p$ and $s_{m}=q$ as $p, q \in X$.

Consider two consecutive points $p_{i}, p_{i+1}$. If $s_{i}=s_{i+1}$, then $\mathrm{d}\left(p_{i}, p_{i+1}\right) \leq 2 \delta$. Otherwise, suppose that $s_{i} \neq s_{i+1}$. If $\mathrm{d}\left(s_{i}, s_{i+1}\right) \leq 1$, then $\pi^{\prime}\left(s_{i}, s_{i+1}\right)=\mathrm{d}\left(s_{i}, s_{i+1}\right) \leq$ $\mathrm{d}\left(p_{i}, p_{i+1}\right)+2 \delta$. If $\mathrm{d}\left(s_{i}, s_{i+1}\right)>1$, then $s_{i}, s_{i+1}$ must be a neighboring pair since $\mathrm{d}\left(p_{i}, p_{i+1}\right) \leq 1$. In this case, it is easy to verify that $\pi^{\prime}\left(s_{i}, s_{i+1}\right) \leq \mathrm{d}\left(p_{i}, p_{i+1}\right)+6 \delta$. Thus,

$$
\begin{aligned}
\pi^{\prime}(p, q) & \leq \sum_{\substack{i=0 \\
m-1}}^{m-1}\left(s_{i}, s_{i+1}\right) \\
& \leq \sum_{i=0}^{m-1} \mathrm{~d}\left(p_{i}, p_{i+1}\right)+6 m \delta \leq \pi(p, q)+6 m \delta
\end{aligned}
$$

Since $p_{0} p_{1} \cdots p_{m}$ is a shortest path, $\mathrm{d}\left(p_{i}, p_{i+2}\right) \geq 1$ for any $0 \leq i \leq m-2$ because otherwise the path could be shortened due to triangular inequality. That is, $\pi(p, q) \geq\lfloor m / 2\rfloor>m / 2-1$, i.e., $m<2(\pi(p, q)+1)$. Thus we have that $\pi^{\prime}(p, q) \leq$ $(1+12 \delta) \pi(p, q)+12 \delta$.

Before we describe the construction of $c$-WSPD for $S$, we need a straightforward extension of geometric well-separated pair decomposition in [10] to two separable point sets.

Lemma 4.10. Suppose that $A$ and $B$ are two point sets that can be separated by a line and have $n$ points in total. For any constant $c \geq 1$, there exists a geometric c-well-separated pair decomposition of $(A, B)$ with $O(n)$ pairs.

Proof. This can be done by modifying the algorithm in [10] so that the first split of the point set of $A \cup B$ is by the line that separates $A$ and $B$.

Now, we describe a process that produces a $c$-WSPD of $S$ for any $c \geq 1$. Set $\delta=1 /(2 c+4)$ and $c^{\prime}=9(c+14)$. We first construct a minimal $\delta$-cover $X$ and the 
set $Z$ as described above. Next we compute a $c^{\prime}$-well-separated pair decomposition of the clusterheads $X$ in the unit-disk graph metric of point set $Z$. Specifically, we give weight 1 to points in $X$ and 0 to bridge points. We find the spanning tree $T$ of the unitdisk graph $I(Z)$. $T$ has total weight $|X|$. We then recursively find balanced weighted decomposition of $T$ : by removing an edge, each subtree has weight at least $1 / \beta$ times the weight of the parent. Since $X$ has a bounded density $O\left(1 / \delta^{2}\right)$, the packing argument is still valid and we can compute a $c^{\prime}$-well-separated pair decomposition for $X$. Suppose the decomposition obtained is $\mathcal{P}=\left\{P_{1}, P_{2}, \ldots, P_{m}\right\}$, where $P_{i}=\left(A_{i}, B_{i}\right)$, $A_{i} \subseteq X, B_{i} \subseteq X$. We now create a set of pairs $\mathcal{P}^{\prime}=\mathcal{P}_{1}^{\prime} \cup \mathcal{P}_{2}^{\prime} \cup \mathcal{P}_{3}^{\prime}$ as follows:

1. For each $P_{i} \in \mathcal{P}$, if $\left|A_{i}\right|>1$ or $\left|B_{i}\right|>1$, we include in $\mathcal{P}_{1}^{\prime}$ the pair $P_{i}^{\prime}=$ $\left(\hat{A}_{i}, \hat{B}_{i}\right)$. Recall that $\hat{A}=\cup_{s \in A} C(s)$.

2. If $\left|A_{i}\right|=\left|B_{i}\right|=1$, suppose that $A_{i}=\{a\}$ and $B_{i}=\{b\}$. If $\mathrm{d}(a, b) \geq(2 c+2) \delta$, we then include in $\mathcal{P}_{1}^{\prime}$ the pair $P_{i}^{\prime}=\left(\hat{A}_{i}, \hat{B}_{i}\right)$. Otherwise, any pair of points in $\hat{A}_{i} \bigcup \hat{B}_{i}$ is within distance $(2 c+2) \delta+2 \delta=1$. Since $\hat{A}_{i} \subset \operatorname{Vor}(a)$, and $\hat{B}_{i} \subset \operatorname{Vor}(b), \hat{A}_{i}$ and $\hat{B}_{i}$ are separable by a line. Per Lemma 4.10 , we compute a geometric $c$-WSPD of $\left(\hat{A}_{i}, \hat{B}_{i}\right)$ and include in $\mathcal{P}_{2}^{\prime}$ all the pairs produced this way.

3. For every $s \in X$, we compute a geometric $c$-WSPD of $C(s)$ and include into $\mathcal{P}_{3}^{\prime}$ all the pairs produced.

Now, we make the next claim.

LEMMA 4.11. $\mathcal{P}^{\prime}$ is a $c$-WSPD of $S$.

Proof. We first argue that $\mathcal{P}^{\prime}$ is a pair decomposition of $S$. For any pair of points $s_{1}, s_{2} \in S$, suppose that the clusterheads covering them are $s_{1}^{\prime}$ and $s_{2}^{\prime}$, respectively. If $s_{1}^{\prime} \neq s_{2}^{\prime}$, then $\left(s_{1}, s_{2}\right)$ is covered by a pair in $\mathcal{P}_{1}^{\prime} \cup \mathcal{P}_{2}^{\prime}$. Otherwise, it is covered by a pair in $\mathcal{P}_{3}^{\prime}$. It is also easily verified that each ordered pair is covered exactly once.

Now, we show that all the pairs in $\mathcal{P}^{\prime}$ are $c$-well-separated with respect to the unit-disk graph metric. Since $\delta=1 /(2 c+4)$, for all the pairs in $\mathcal{P}_{2}^{\prime}$, the Euclidean distance between any two points in $\hat{A}_{i} \cup \hat{B}_{i}$ is at most $(2 c+4) \delta=1$. Therefore, the unit-disk graph on the subset $\hat{A}_{i} \cup \hat{B}_{i}$ is a complete graph, i.e., every pair in $\mathcal{P}_{2}^{\prime}$ is $c$-well-separated under the unit-disk graph metric. The same argument applies to $\mathcal{P}_{3}^{\prime}$ as the distance between two points in $C(s)$ is at most $2 \delta \leq 1$.

Now, consider a pair $\left(\hat{A}_{i}, \hat{B}_{i}\right) \in \mathcal{P}_{1}^{\prime}$. We distinguish two cases:

1. When $\left|A_{i}\right|=\left|B_{i}\right|=1$. Then we must have $\pi\left(A_{i}, B_{i}\right) \geq(2 c+2) \delta$ according to the construction rule, and thus

$$
\pi\left(\hat{A}_{i}, \hat{B}_{i}\right) \geq \pi\left(A_{i}, B_{i}\right)-2 \delta \geq 2 c \delta=c /(c+2) .
$$

On the other hand, $D\left(\hat{A}_{i}\right), D\left(\hat{B}_{i}\right) \leq 2 \delta=1 /(c+2)$. Therefore, $\left(\hat{A}_{i}, \hat{B}_{i}\right)$ is $c$-wellseparated.

2. When $\left|A_{i}\right|>1$ or $\left|B_{i}\right|>1$. In what follows, we use $D$ and $D^{\prime}$ to denote $D_{\pi}, D_{\pi^{\prime}}$, respectively. Clearly,

$$
\pi\left(\hat{A}_{i}, \hat{B}_{i}\right) \geq \pi\left(A_{i}, B_{i}\right)-2 \delta, \text { and } D(\hat{A}) \leq D(A)+2 \delta .
$$

Since either $A_{i}$ or $B_{i}$ contains at least two clusterheads, it must be true that $\max \left(D\left(A_{i}\right), D\left(B_{i}\right)\right) \geq \delta$, as the distance between two clusterheads is at least $\delta$. So, $\max \left(D\left(\hat{A}_{i}\right), D\left(\hat{B}_{i}\right)\right) \geq \delta$, and $\max \left(D\left(\hat{A}_{i}\right), D\left(\hat{B}_{i}\right)\right) \leq \max \left(D\left(A_{i}\right), D\left(B_{i}\right)\right)+2 \delta \leq$ $3 \max \left(D\left(A_{i}\right), D\left(B_{i}\right)\right)$.

As $A_{i}, B_{i}$ are $c^{\prime}$-well-separated under $\pi^{\prime}, \pi^{\prime}\left(A_{i}, B_{i}\right) \geq c^{\prime} \cdot \max \left(D^{\prime}\left(A_{i}\right), D^{\prime}\left(B_{i}\right)\right)$. 
Therefore,

$$
\begin{aligned}
\pi\left(\hat{A}_{i}, \hat{B}_{i}\right) \geq & \pi\left(A_{i}, B_{i}\right)-2 \delta \\
\geq & \left(\pi^{\prime}\left(A_{i}, B_{i}\right)-12 \delta\right) /(1+12 \delta)-2 \delta \\
& \text { by Lemma } 4.9 \\
\geq & c^{\prime} /(1+12 \delta) \cdot \max \left(D^{\prime}\left(A_{i}\right), D^{\prime}\left(B_{i}\right)\right)-14 \delta \\
\geq & c^{\prime} /(1+12 \delta) \cdot \max \left(D\left(A_{i}\right), D\left(B_{i}\right)\right)-14 \delta \\
\geq & \left(c^{\prime} /(3(1+12 \delta))-14\right) \cdot \max \left(D\left(A_{i}\right), \hat{D}\left(B_{i}\right)\right) \\
\geq & c \max \left(D\left(\hat{A}_{i}\right), D\left(\hat{B}_{i}\right)\right) . \\
& \quad \text { by } c \geq 1, \delta=1 /(2 c+4), \text { and } c^{\prime}=9(c+14) .
\end{aligned}
$$

In both cases, $\hat{A}_{i}, \hat{B}_{i}$ are $c$-well-separated, i.e., all the pairs in $\mathcal{P}_{1}^{\prime}$ are $c$-wellseparated.

Now, we make the claim in the next theorem.

THEOREM 4.12. For any set $S$ of $n$ points in the plane and any $c \geq 1$, there exists a $c$-WSPD $\mathcal{P}$ of $S$ under the unit-disk graph metric where $\mathcal{P}$ contains $O\left(c^{4} n \log n\right)$ pairs and can be computed in $O\left(c^{4} n \log n\right)$ time.

Proof. By combining Corollary 4.6 and Lemma 4.8, we have that $\left|\mathcal{P}_{1}^{\prime}\right| \leq|\mathcal{P}|=$ $O\left(c^{2} n \log n / \delta^{2}\right)=O\left(c^{4} n \log n\right)$. If $\left|A_{i}\right|=1$, then the number of pairs $\left(A_{i}, B_{i}\right) \in \mathcal{P}_{2}^{\prime}$ where $\left|B_{i}\right|=1$ is bounded by $O\left(1 / \delta^{2}\right)=O\left(c^{2}\right)$. Since the size of the geometric well-separated pair decomposition is linear in terms of the number of points [10], $\left|\mathcal{P}_{2}^{\prime}\right|=O\left(c^{2} n\right)$. Clearly, $\left|\mathcal{P}_{3}^{\prime}\right|=O(n)$. When we sum the sizes, we have that $\left|\mathcal{P}^{\prime}\right|=$ $O\left(c^{4} n \log n\right)$.

By Theorem 4.5 and Lemma 4.8, it is easy to see that the total time needed is $O\left(c^{4} n \log n\right)$.

Similarly, in higher dimensions, we have the next corollary.

Corollary 4.13. For any set $S$ of $n$ points in $\mathbb{R}^{k}$, for $k \geq 3$, and for any constant $c \geq 1$, there exists a $c$-WSPD $\mathcal{P}$ of $S$ under the unit-ball graph metric where $\mathcal{P}$ contains $O\left(n^{2-2 / k}\right)$ pairs and can be constructed in $O\left(n^{4 / 3}\right.$ polylog $\left.n\right)$ time for $k=3$ and in $O\left(n^{2-2 / k}\right)$ time for $k \geq 4$.

Proof. For simplicity of computation, we use boxes instead of balls to find clusterheads with constant bounded density. A point $p$ is covered by a point $s$ if $p$ is inside the box with size $2 \delta$ centered at $s$. Finding the minimal cover can be done by using a dynamic rectilinear range search tree in $k$-dimensions [11]. The running time is $O(n$ polylog $n)$. Notice that every point can be covered by at most a constant number of clusterheads; thus we can find the nearest clusterhead for every point in linear time in total. To find a bridge between two clusterheads $s_{1}, s_{2}$, we compute the bichromatic closest pair between two sets $C\left(s_{1}\right), C\left(s_{2}\right)$. Let $m_{1}=\left|C\left(s_{1}\right)\right|$ and $m_{2}=\left|C\left(s_{2}\right)\right|$. According to [1], when $k=3$, it takes $O\left(\left(m_{1} m_{2}\right)^{2 / 3}\right.$ polylog $\left.n\right)$ time, and when $k=4$, it is

$$
\begin{aligned}
& O\left(\left(m_{1} m_{2}\right)^{1-1 /(\lceil k / 2\rceil+1)+\varepsilon}+m_{1} \log m_{2}+m_{2} \log m_{1}\right) \\
= & O\left(\left(m_{1} m_{2}\right)^{1-1 / k}+m_{1} \log m_{2}+m_{2} \log m_{1}\right) .
\end{aligned}
$$

Since each set is involved only in $O(1)$ bichromatic closest pair computation, the total time is $O\left(n^{4 / 3} \operatorname{polyl} \log n\right)$ when $k=3$ and $O\left(n^{2-2 / k}\right)$ for $k \geq 4$. Computing the WSPD on the clusterheads takes $O\left(n^{2-2 / k}\right)$ time, according to Theorem 4.5.

4.3. Estimating distance between pairs. In the above, we showed how to construct well-separated pair decomposition for unit-disk and unit-ball graphs. As mentioned in the introduction, to apply WSPD in solving proximity problems in 
the unit-disk graphs, we first need to estimate the shortest path distances between $O(n \log n)$ pairs of the WSPD. Note that in our construction for the point sets with constant bounded density, we use Euclidean distance as a lower bound for the unitdisk graph distance and the size of the point set as an upper bound for the diameter. While these approximations are sufficient for bounding the size of WSPDs, they are too coarse for obtaining good approximation. Recall that $\sigma(A)$ is an (arbitrary) point picked from a set $A$. For a $c$-well-separated pair $(A, B)$, we can use the estimated distance $\hat{\pi}(\sigma(A), \sigma(B))$ to approximate all the pairwise distances between points in $A$ and points in $B$. In this section, we show several trade-offs for measuring the distance between $m$ pairs of points in the unit-disk graph.

Denote by $\tau(n, c, m)$ the time needed to compute $m$-pairs $c$-approximate distance in a unit disk graph. In what follows, we set $c_{0}=2.42>\frac{4 \sqrt{3}}{9} \pi$ and $c_{1}$ a number slightly smaller than $c_{0}$ but greater than $\frac{4 \sqrt{3}}{9} \pi$.

LEMMA 4.14.

1. $\tau\left(n, c_{1}, m\right)=O\left(n \log ^{3} n+m\right)$.

2. $\tau(n, 1+\varepsilon, m)=O\left(n^{2} /(\varepsilon r)+m r / \varepsilon\right)$, for any $1 \leq r \leq n$.

Proof. 1. We first construct a planar $\frac{4 \sqrt{3}}{9} \pi$-spanner of the unit disk graph. Such spanner exists and can be computed in $O(n \log n)$ time [26]. Now, we apply Thorup's construction of $(1+\varepsilon)$-approximate distance oracle [35] to that planar spanner, for a sufficiently small constant $\varepsilon>0$. The bound follows immediately from the preprocessing and query time bounds of Thorup's algorithm.

2. We again cluster the points and consider the set of clusterheads, $X$. Suppose that we have constructed a $(1+\varepsilon / 2)$-approximate shortest distance oracle for $X$. For two query points $q_{1}, q_{2}$, if $\mathrm{d}\left(q_{1}, q_{2}\right) \leq 1$, we return $\mathrm{d}\left(q_{1}, q_{2}\right)$. Otherwise, we find the clusterheads $s_{1}, s_{2}$ that cover $q_{1}$ and $q_{2}$, respectively, and return $\hat{\pi}\left(q_{1}, q_{2}\right)=$ $\pi^{\prime}\left(s_{1}, s_{2}\right)+2 \delta$ as an approximation of $\pi\left(q_{1}, q_{2}\right)$. It is easily verified that $\hat{\pi}\left(q_{1}, q_{2}\right)$ is a $(1+\varepsilon)$-approximation for $\delta=O(\varepsilon)$. The density of $X$ is $O\left(1 / \delta^{2}\right)=O\left(1 / \varepsilon^{2}\right)$. The graph formed by connecting neighboring pairs in $X$ is an $O\left(1 / \varepsilon^{2}\right)$-overlap graph as defined by Miller, Teng, and Vavasis [28] and therefore admits a balanced separator with size $O(\sqrt{n} / \varepsilon)$. Furthermore, it can be computed in deterministic linear time by the method of Eppstein, Miller, and Teng [13].

Now, it is easy to extend the shortest distance algorithm for planar graphs by Arikati et al. [3] to the above geometric graph on $X$. By using the same technique, we can obtain a trade-off with $O\left(n^{2} /(\varepsilon r)\right)$ preprocessing time and $O(r / \varepsilon)$ query time for any $1 \leq r \leq \sqrt{n}$.

5. Applications. In this section, we show the application of the well-separated pair decomposition in obtaining efficient algorithms for approximating the furthest neighbor (diameter, center), nearest neighbor (closest pair), median, and stretch factor, all under the unit-disk graph metric. Since the running time of the algorithms for computing $c_{0}$-approximate and $(1+\varepsilon)$-approximate distance are different, we will be describing the bounds for both approximations (recall that $c_{0}=2.42$ ). Roughly speaking, our algorithms for computing $c_{0}$-approximation is about linear and for computing $(1+\varepsilon)$-approximation is about $O(n \sqrt{n})$, dominated by the distance estimation.

We should note that for the problems of computing diameter and center, there is a simple linear time method to achieve 2-approximation. It is therefore not interesting to present algorithms to obtain $c_{0}$-approximation for those problems, with $c_{0}=2.42$. For the other problems, it is still interesting, as we are not aware of any algorithms that achieve comparable approximation ratio in subquadratic time, even for planar graphs. 
We need first to describe the well-separated pair decomposition we will be using. In what follows, we also include the time for measuring the distances between pairs into the construction time. For $c_{0}$-approximation, $c_{0}=2.42$, we construct a $c$-wellseparated pair decomposition $\mathcal{P}_{1}$ for sufficiently large constant $c$ and, for each pair $(A, B)$ in the WSPD, compute $c_{1}$-approximate distance $\hat{\pi}_{1}(A, B)$ between $\sigma(A)$ and $\sigma(B)$ according to Lemma 4.14.1. For $(1+\varepsilon)$-approximation, we compute a $c$-wellseparated pair decomposition $\mathcal{P}_{2}$ for $c=O(1 / \varepsilon)$ and, for each pair $(A, B)$, compute the $(1+\varepsilon / 2)$-approximate distance $\hat{\pi}_{2}(A, B)$ between $(\sigma(A), \sigma(B))$ by Lemma 4.14 .2 and by setting $r=\varepsilon^{2} \sqrt{n / \log n}$. The following is immediate.

LEMMA 5.1. $\mathcal{P}_{1}$ contains $O(n \log n)$ pairs and can be computed in $O\left(n \log ^{3} n\right)$ time. $\mathcal{P}_{2}$ contains $O\left(n \log n / \varepsilon^{4}\right)$ pairs and can be computed in $O\left(n \sqrt{n \log n} / \varepsilon^{3}\right)$ time. For any pair of points $(p, q)$, suppose that its covering pair in $\mathcal{P}_{1}\left(\mathcal{P}_{2}\right)$ is $(A, B)$; then $\hat{\pi}_{1}(A, B)\left(\hat{\pi}_{2}(A, B)\right)$ is a c co-approximation $\left((1+\varepsilon)\right.$-approximation) of $\pi(p, q), c_{0}=$ 2.42 .

In the process of producing a well-separated pair decomposition, we constructed several trees, the balanced hierarchical decomposition tree for constant bounded density points and the fair split trees for geometric well-separated pair decomposition [10]. For simplicity of presentation, we treat them as a single tree $T_{1}^{\prime}$ and $T_{2}^{\prime}$, for $\mathcal{P}_{1}$ and $\mathcal{P}_{2}$, respectively, by joining the trees created in the geometric well-separated pair decomposition to the clusterheads appropriately. In what follows, $\mathcal{P}, T^{\prime}, \hat{\pi}$ mean that they could be either case.

5.1. $(1+\varepsilon)$-distance oracle. Although $\mathcal{P}_{2}$ takes time $O\left(n \sqrt{n \log n} / \varepsilon^{3}\right)$, the space needed is only $O\left(n \log n / \varepsilon^{4}\right)$. We can use $\mathcal{P}_{2}$ to answer $(1+\varepsilon)$-approximate distance query between any two points $\left(p_{1}, p_{2}\right)$ by first locating the pair $(A, B)$ that covers $(p, q)$ and returning $\hat{\pi}(A, B)$. The query time is the time needed to discover a pair in $\mathcal{P}_{2}$ that covers the query pair. We show that this can be done in $O(1)$ time by using the properties of WSPD.

COROLlary 5.2. For a unit-disk graph on $n$ points and for any $\varepsilon>0$, we can preprocess it into a data structure with $O\left(n \log n / \varepsilon^{4}\right)$ size so that for any query pair, $a(1+\varepsilon)$-approximate distance can be answered in $O(1)$ time.

Proof. It suffices to prove for constant-bounded density point sets. We store all the pairs in $\mathcal{P}$ in a hash table indexed by the pairs. We will show that for each query pair $(p, q)$, we can find $O(1)$ candidate pairs that are guaranteed to contain the pair in $\mathcal{P}$ that covers $(p, q)$. Then, we simply query the hash table using those candidate pairs and discover the one that does cover $(p, q)$.

We modify our construction in section 4.1 so that we are more careful on deciding when to include a pair in $\mathcal{P}$. We use a $c_{1}$-approximate distance oracle as constructed in Lemma 4.14.1. When producing $\mathcal{P}$, we include a pair in $\mathcal{P}$ if $\hat{\pi}(A, B)>\left(c c_{1}+\right.$ $2) \max (|A|-1,|B|-1)$. Then there is a constant $c_{2}>0$ such that for any $c \geq 2$ and any pair $(A, B) \in \mathcal{P}, c c_{1} s \leq \pi(A, B) \leq c c_{2} s$, where $s=\max (|A|-1,|B|-1)$.

Now, to answer a query $(p, q)$, we first use the $c_{1}$-approximate distance oracle to compute an approximation $\ell$ of $\pi(p, q)$, i.e., $\pi(p, q) \leq \ell \leq c_{1} \pi(p, q)$. Suppose that $(A, B) \in \mathcal{P}$ is the pair that covers $(p, q)$. Without loss of generality, let us assume that $|A| \geq|B|$, i.e., $s=|A|-1$. Then we have

$$
s \leq \pi(A, B) /\left(c c_{1}\right) \leq \pi(p, q) /\left(c c_{1}\right) \leq \ell /\left(c c_{1}\right)
$$

On the other hand, $s \geq \pi(A, B) /\left(c c_{2}\right) \geq(\pi(p, q)-2 s) /\left(c c_{2}\right)$. That is, $s \geq$ $\pi(p, q) /\left(c c_{2}+2\right) \geq \ell /\left(c_{1}\left(c c_{2}+2\right)\right)$. 
Set $\hat{\ell}=\ell /\left(c c_{1}\right)$. Then, for $(A, B)$ to cover $(p, q), A$ has to be an ancestor of $p$ in $T^{\prime}$, and the size of $A$ is sandwiched by $\hat{\ell} /\left(c_{2}+1\right)$ and $\hat{\ell}$. Notice that $c_{1}, c_{2}$ are constants independent of $c$. There are only $O(1)$ such nodes in $T^{\prime}$. Similarly, there are only $O(1)$ such $B$ 's. We now form $O(1)$ candidate pairs by joining every pair. Clearly, this can be done in $O(1)$ time.

5.2. Furthest neighbor. Suppose that $S_{1} \subseteq S$. For any $p$, define the (relative) furthest neighbor of $p$ to be $\xi(p)=\arg \max _{q \in S_{1}} \pi(p, q)$ in $S_{1}$. Then the diameter of $S_{1}$ is $D\left(S_{1}\right)=\max _{p \in S_{1}} \pi(p, \xi(p))$. The center of $S_{1}$ is the point that minimizes the maximum distance to the other points, i.e., $\arg \min _{p \in S_{1}} \pi(p, \xi(p))$. Therefore, once we compute approximate furthest neighbors for all the $p$, we also obtain approximate diameter and center.

Consider any WSPD. To compute the furthest neighbor of $S_{1}$, we traverse the balanced hierarchical decomposition tree $T^{\prime}$ and mark all the nodes $v \in T^{\prime}$, where $S(v) \cap S_{1} \neq \emptyset$. This can be done in $O(n)$ time in a postorder visit of the tree. A pair $P=(S(u), S(v))$ is called marked if both $u$ and $v$ are marked. Let

$$
R_{1}(u)=\max \{\hat{\pi}(S(u), B) \mid(S(u), B) \text { is marked }\}
$$

and 0 if there is no such pair. With each node $u$, we also record $\ell(u)$, the node that achieves $R_{1}(u)$.

For any $p \in S_{1}$, consider the path $P$ in $T^{\prime}$ from $p$ to the root. Suppose that $u$ is the node that maximizes $R_{1}(u)$ among all the nodes on $P$. Now, we pick any point, say, $q$, from $S(\ell(u)) \cap S_{1}$ (since $\ell(u)$ is marked, $S(\ell(u)) \cap S_{1} \neq \emptyset$ ) and claim that it is an approximate furthest neighbor with the approximation ratio 2.42 , if the above process is applied to $\mathcal{P}_{1}$, or $1+\varepsilon$, if applied to $\mathcal{P}_{2}$. For correctness, consider the (marked) pair in $\mathcal{P}$ that covers $(p, \xi(p))$. Suppose it is $(S(u), S(v))$. Then $R_{1}(u) \geq \hat{\pi}(S(u), S(v))$. Since the pairs are well-separated, it is easy to see that $q$ is an approximate furthest neighbor of $p$ with the approximation ratio determined by the WSPD we use. After we have computed the approximate furthest neighbor, it is simple to compute the diameter and the center. Therefore, we have the next corollary.

Corollary 5.3. For any set $S$ of $n$ points in the plane and any $S_{1} \subseteq S$, we can compute

- $c_{0}$-approximate furthest neighbor for all the points in $S_{1}$ in $O\left(n \log ^{3} n\right)$ time, $c_{0}=2.42 ;$ and

- $(1+\varepsilon)$-approximation, for any $\varepsilon>0$, of the furthest neighbor, the diameter of $S_{1}$, and the center of $S_{1}$ in $O\left(n \sqrt{n \log n} / \varepsilon^{3}\right)$ time.

Remark. We did not list $c_{0}$-approximation $\left(c_{0}=2.42\right)$ for the diameter and the center because there is a simple linear time 2-approximate algorithm.

5.3. Nearest neighbor, closest pair. Computing the nearest neighbor or closest pair in $S$ under the unit-disk graph metric is trivial-it is the same as under the Euclidean metric as long as the graph is connected. However, the problem becomes harder if we restrict our attention to a subset $S_{1} \subseteq S$, i.e., computing the nearest neighbor in $S_{1}$ for each point in $S_{1}$ or computing the closest pair between points in $S_{1}$. For any two sets $S_{1}, S_{2}$, we can also define the bichromatic closest pair to be $\arg \min _{p \in S_{1}, q \in S_{2}} \pi\left(S_{1}, S_{2}\right)$.

By using the same technique as in the previous section, we are able to show the next corollary.

Corollary 5.4. For any set $S$ of $n$ points in the plane, and any $S_{1}, S_{2} \subseteq S$, we can compute 
- $c_{0}$-approximation $\left(c_{0}=2.42\right)$ for the nearest neighbor for all the points in $S_{1}$, the closest pair in $S_{1}$, the bichromatic closest pair of $S_{1}, S_{2}$, in time $O\left(n \log ^{3} n\right) ;$ and

- $(1+\varepsilon)$-approximation for the same problems in time $O\left(n \sqrt{n \log n} / \varepsilon^{3}\right)$.

Remark. We should note that by applying the technique in [10], we can actually enumerate a set of $O(n)$ pairs of points that is guaranteed to include the closest pair. However, since our distance oracle is approximate, we can compute only the approximate closest pair, unlike in the geometric case.

5.4. Median. Similar to the definition of center, median is defined to be the point that minimizes the average (or total) distance to all the other points. Let $\rho(p)=\sum_{q \in S_{1}} \pi(p, q)$. Then the median of $S_{1}$ is the point that minimizes $\rho(p)$.

By using a similar technique, we can show the following.

Corollary 5.5. For any planar point set $S$ with $n$ points and $S_{1} \subseteq S, a c_{0}$ approximate median ( $\left.c_{0}=2.42\right)$ of $S_{1}$ can be computed in $O\left(n \log ^{3} n\right)$ time, and for any $\varepsilon>0$, a $(1+\varepsilon)$-approximation can be computed in $O\left(n \sqrt{n \log n} / \varepsilon^{3}\right)$ time.

Proof. Computing approximate median is similar to computing the furthest neighbor. The only difference is that instead of computing $R_{1}(u)$, we compute

$$
R_{2}(u)=\sum_{(S(u), B) \in \mathcal{P}} \hat{\pi}(S(u), B) \cdot|B|
$$

and then for each point $p$ and the path $P$ from $p$ to the root, compute $\hat{\rho}(p)=$ $\sum_{u \in P} R_{2}(u) /(n-1)$, as an approximation of $\rho(p)$. The correctness is guaranteed by the property of pair decomposition that every pair of points is covered by a unique pair in the decomposition. Again, we pick the point with the minimum $\hat{\rho}(p)$ to be the approximate median. The approximation ratio and running time bounds follow immediately.

5.5. Stretch factor. For a graph $G$ defined on $S$, the stretch factor of $G$ with respect to $\pi$ is defined as $\max _{p, q \in S} \pi_{G}(p, q) / \pi(p, q)$. Narasimhan and Smid [29] gave an algorithm to approximate the stretch factor of a geometric graph to the Euclidean metric using the geometric well-separated pair decomposition. By following the same argument we can approximate the stretch factor of an arbitrary graph $G$ with respect to the unit-disk graph metric. Again, we consider the well-separated pair decomposition $\mathcal{P}$. For each pair $(A, B) \in \mathcal{P}$, we pick any pair of points $(p, q)$, where $p \in A$ and $q \in B$, and compute the approximate shortest path $\hat{\pi}_{G}(p, q)$ in $G$ and $\hat{\pi}(p, q)$ in $I$. The maximum ratio of $\hat{\pi}_{G}(p, q) / \hat{\pi}(p, q)$ over all pairs in $\mathcal{P}$ is an approximation to the stretch factor by the same argument in [29].

Corollary 5.6. For any graph $G$ on $S$, we can compute an $O(1)$-approximate stretch factor of $G$ in time $O\left(\tau_{1}^{\prime}(n \log n)\right)$, where $\tau_{1}^{\prime}(m)$ is the time to compute $m$ $O(1)$-approximate shortest path queries in $G$. In particular, if $G$ is a subgraph of $I$, an $O(1)$-approximate can be computed in time $O\left(n \log ^{3} n\right)$. Similarly, we can compute for any $\varepsilon>0, a(1+\varepsilon)$-approximate stretch factor of $G$ in time $O\left(\tau_{2}^{\prime}\left(n \log n / \varepsilon^{4}\right)+\right.$ $\left.n \sqrt{n \log n} / \varepsilon^{3}\right)$, where $\tau_{2}^{\prime}(m)$ is the time to compute $m(1+\varepsilon)$-approximate shortest path queries in $G$. When $G$ is a subgraph of $I$, this can be done in $O\left(n \sqrt{n \log n} / \varepsilon^{3}\right)$ time.

6. Extensions. There are several direct extensions of our techniques. Here, we outline the extension to the intersection graph of disks with bounded radii ratio and to the unweighted unit-disk graph. 
6.1. Intersection graphs of disks with bounded radii ratio. When the sizes of the disks are not uniform, it is generally not possible to obtain subquadratic well-separated pair decomposition of the metric induced by the intersection graph. This can be shown by the example where there is a big disk and $n-1$ pairwise disjoint small disks intersecting it. Indeed, the intersection graph of this example is a tree with one internal node and $n-1$ leaves.

However, if the ratio between the radii of any two disks (or balls) is upper bounded by a constant, then the packing property (Lemma 4.3) still holds. We can obtain similar results for the intersection graph of disks (or balls in high dimensions) with bounded radii ratio.

6.2. Unweighted unit-disk graphs. In the previous part, we considered only weighted unit-disk graphs. There are applications in which we need the unweighted unit-disk graph. The results for point set with constant bounded density can be directly extended to unweighted unit-disk graphs. If the density is unbounded, then it is impossible to obtain a subquadratic size well-separated pair decomposition as shown by the example of the unweighted complete graph. But again for the applications, we can apply the clustering technique to reduce it to the problem for point sets with constant unbounded density. The clustering increases the approximation ratio by a multiplicative factor of 3 [17]. Thus in near linear time we can compute $3 c_{0^{-}}$ approximation $\left(c_{0}=2.42\right)$ for the following problems: the furthest neighbor, nearest neighbor, closest pair, bichromatic closest pair, median, and stretch factor, all with respect to the unweighted unit-disk graph metric. Again we didn't list the problems of computing diameter and center because there are trivial 2-approximate algorithms.

Furthermore, we can get a better multiplicative approximation factor by permitting an additive error as shown in the following. For the unweighted unit-disk graph $I(S)$ on point set $S$, we cluster the points by finding a minimal 1-cover $X$ of $S$; i.e., any two clusterheads $c_{1}, c_{2} \in X$ must be distance at least 1 away, and any point is covered by at least one clusterhead. We also assign a unique clusterhead $c(p)$ to every node $p$ in $S$, as before. For any two clusterheads in $X$ within distance 3 , if there exists a path with no more than three hops to connect them, we select such two nodes as a bridge. Define $Z$ to be the union of centers $X$ and bridge nodes $Y$. Again, the shortest path metric in the unweighted unit-disk graph $I(Z)$ is denoted by $\pi^{\prime}$, to be distinguished by the metric $\pi$ in $I(S)$. It is easy to see that $Z$ has constant bounded density. So we build the $c$-well-separated pair decomposition $\mathcal{P}^{\prime}$ on $Z$. For each pair $\left(A^{\prime}, B^{\prime}\right) \in \mathcal{P}^{\prime}$, we build a pair $(A, B)$, where $A=\bigcup_{c(p) \in A^{\prime}} p, B=\bigcup_{c(q) \in B^{\prime}} q$. The collection of the pairs is denoted by $\mathcal{P}$.

Lemma 6.1. For $p, q \in S$,

1. $\pi(p, q) \leq \pi^{\prime}(p, q)$;

2. $\pi^{\prime}(p, q) \leq 3 \pi(p, q)+2$, if $p, q$ are clusterheads, then $\pi^{\prime}(p, q) \leq 3 \pi(p, q)$.

Proof. The first claim is because $I(Z)$ is a subgraph of $I(S)$. The second one is proved in [17].

Set $c=6 / \varepsilon$; we have the next lemma.

Lemma 6.2. For any two pairs of points $\left(p_{1}, q_{1}\right),\left(p_{2}, q_{2}\right) \in(A, B)$, where $(A, B) \in$ $\mathcal{P}, \pi\left(p_{1}, q_{1}\right) \leq(1+\varepsilon) \pi\left(p_{2}, q_{2}\right)+(4+2 \varepsilon)$.

Proof. Take the centers of $p_{2}, q_{2}, c\left(p_{2}\right) \in A^{\prime}, c\left(q_{2}\right) \in B^{\prime}$. We can see that $\pi\left(p_{1}, c\left(p_{2}\right)\right) \leq 1+\pi\left(c\left(p_{1}\right), c\left(p_{2}\right)\right) \leq 1+\pi^{\prime}\left(c\left(p_{1}\right), c\left(p_{2}\right)\right) \leq 1+D^{\prime}\left(A^{\prime}\right)$, where $D^{\prime}$ denote the diameter in metric $\pi^{\prime}$. Since $\left(A^{\prime}, B^{\prime}\right)$ is $c$-well-separated, we have $\pi^{\prime}\left(A^{\prime}, B^{\prime}\right) \geq$ $c \cdot \max \left(D^{\prime}\left(A^{\prime}\right), D^{\prime}\left(B^{\prime}\right)\right)$. So $\pi^{\prime}\left(c\left(p_{2}\right), c\left(q_{2}\right)\right) \geq c \cdot \max \left(D^{\prime}\left(A^{\prime}\right), D^{\prime}\left(B^{\prime}\right)\right) \cdot \pi\left(c\left(p_{2}\right), c\left(q_{2}\right)\right) \leq$ 
$\pi\left(p_{2}, q_{2}\right)+2$. Combining Lemma 6.1 and all these, we have

$$
\begin{aligned}
\pi\left(p_{1}, q_{1}\right) & \leq \pi\left(p_{1}, c\left(p_{2}\right)\right)+\pi\left(c\left(p_{2}\right), c\left(q_{2}\right)\right)+\pi\left(c\left(q_{2}\right), q_{1}\right) \\
& \leq 1+D^{\prime}\left(A^{\prime}\right)+\pi\left(c\left(p_{2}\right), c\left(q_{2}\right)\right)+1+D^{\prime}\left(B^{\prime}\right) \\
& \leq \pi\left(c\left(p_{2}\right), c\left(q_{2}\right)\right)+2+(2 / c) \pi^{\prime}\left(c\left(p_{2}\right), c\left(q_{2}\right)\right) \\
& \leq \pi\left(c\left(p_{2}\right), c\left(q_{2}\right)\right)+2+(2 / c)\left(3 \pi\left(c\left(p_{2}\right), c\left(q_{2}\right)\right)\right) \\
& \leq(1+6 / c) \pi\left(c\left(p_{2}\right), c\left(q_{2}\right)\right)+2 \\
& \leq(1+6 / c) \pi\left(p_{2}, q_{2}\right)+(4+12 / c) \\
& =(1+\varepsilon) \pi\left(p_{2}, q_{2}\right)+(4+2 \varepsilon) .
\end{aligned}
$$

THEOREM 6.3. For an unweighted unit-disk graph $I(S)$ on a point set $S$ and any $1>\varepsilon>0$, we can find in time $O\left(n \sqrt{n \log n} / \varepsilon^{3}\right)$ a data structure of size $O\left(n \log n / \varepsilon^{4}\right)$ such that for any pair of points $p, q$ with distance $\pi(p, q)>2$, we can return a value $x$ in $O(1)$ time such that

$$
\frac{1}{1+\varepsilon}(x-4-2 \varepsilon) \leq \pi(p, q) \leq(1+\varepsilon) x+4+2 \varepsilon .
$$

Proof. For each pair $\left(A^{\prime}, B^{\prime}\right)$, we take an arbitrary pair of points $a_{0}, b_{0}$ from $A^{\prime}, B^{\prime}$, respectively, and compute the distance $\pi\left(a_{0}, b_{0}\right)$. By using the same idea as in Lemma 4.14, we can show that the total amount of time is $O\left(n \sqrt{n \log n} / \varepsilon^{3}\right)$. Note that any pair of points $p, q$ with distance $\pi(p, q)>2$ must be in different clusters. We find the pair $\left(A^{\prime}, B^{\prime}\right)$ that includes the pair of points $(c(p), c(q))$ in $O(1)$ time. (Notice that there exists a constant-spanner for the unweighted unit-disk graph on point set with constant density [17].) Therefore we take $x=\pi\left(a_{0}, b_{0}\right)$, where $\left(a_{0}, b_{0}\right)$ is the representative pair of $\left(A^{\prime}, B^{\prime}\right)$. The theorem then follows from Lemma 6.2.

Similarly, this gives us $O\left(n \sqrt{n \log n} / \varepsilon^{3}\right)$-time algorithms for finding approximate solutions to the following problems: the furthest neighbor, nearest neighbor, closest pair, bichromatic closest pair, median, diameter, center, and stretch factor, all with respect to the unweighted unit-disk graph metric.

7. Conclusion. In this paper, we extend the well-separated pair decomposition, originally developed in the Euclidean metric, to the unit-disk and unit-ball graph metrics. This allows us to obtain almost linear time 2.42-approximate and subquadratic time $(1+\varepsilon)$-approximate algorithms for several proximity problems where no efficient methods were previously known. The combinatorial bounds in $\mathbb{R}^{k}$ for $k \geq 3$ are also tight.

The most notable open problem is the gap between $\Omega(n)$ and $O(n \log n)$ on the number of pairs needed in the plane. Also, the time bound for $(1+\varepsilon)$-approximation is still about $\widetilde{O}(n \sqrt{n})$ because of the lack of efficient method for computing $(1+\varepsilon)$ approximate shortest distance between $O(n)$ pairs of points. Any improvement to the algorithm for that problem will immediately lead to improvement to all the $(1+\varepsilon)$ approximate algorithms presented in this paper.

\section{REFERENCES}

[1] P. K. Agarwal, H. Edelsbrunner, O. Schwarzkopf, and E. Welzl, Euclidean minimum spanning trees and bichromatic closest pairs, Discrete Comput. Geom., 6 (1991), pp. 407422.

[2] D. Aingworth, C. Chekuri, and R. Motwani, Fast estimation of diameter and shortest paths (without matrix multiplication), in Proceedings of the 7th ACM-SIAM Symposium on Discrete Algorithms, SIAM, Philadelphia, 1996, pp. 547-553. 
[3] S. R. Arikati, D. Z. Chen, L. P. Chew, G. Das, M. H. M. Smid, and C. D. Zaroliagis, Planar spanners and approximate shortest path queries among obstacles in the plane, in Proceedings of the 4th Annual European Symposium on Algorithms, J. Díaz and M. Serna, eds., 1996, pp. 514-528.

[4] S. Arya, G. Das, D. M. Mount, J. S. Salowe, and M. Smid, Euclidean spanners: Short, thin, and lanky, in Proceedings of the 27th ACM Symposium on Theory of Computing, 1995, pp. 489-498.

[5] S. ARYA, D. M. Mount, AND M. SMID, Randomized and deterministic algorithms for geometric spanners of small diameter, in Proceedings of the 35th IEEE Symposium on Foundations of Computer Science, 1994, pp. 703-712.

[6] H. Breu AND D. G. KirkPatrick, Unit disk graph recognition is NP-hard, Computational Geom., 9 (1998), pp. 3-24.

[7] Callahan and Kosaraju, Faster algorithms for some geometric graph problems in higher dimensions, in Proceedings of the 4th ACM-SIAM Symposium on Discrete Algorithms, SIAM, Philadelphia, 1993, pp. 291-300.

[8] P. B. Callahan, Optimal parallel all-nearest-neighbors using the well-separated pair decomposition, in Proceedings of the 34th IEEE Symposium on Foundations of Computer Science, 1993, pp. 332-340.

[9] P. B. Callahan and S. R. Kosaraju, Algorithms for dynamic closest-pair and n-body potential fields, in Proceedings of the 6th ACM-SIAM Symposium on Discrete Algorithms, SIAM, Philadelphia, 1995, pp. 263-272.

[10] P. B. Callahan and S. R. Kosaraju, A decomposition of multidimensional point sets with applications to $k$-nearest-neighbors and n-body potential fields, J. ACM, 42 (1995), pp. 6790.

[11] Y.-J. Chiang and R. Tamassia, Dynamic algorithms in computational geometry, Proc. IEEE, 80 (1992), pp. 1412-1434.

[12] B. N. Clark, C. J. Colbourn, and D. S. Johnson, Unit disk graphs, Discrete Math., 86 (1990), pp. 165-177.

[13] D. Eppstein, G. L. Miller, And S.-H. Teng, A deterministic linear time algorithm for geometric separators and its applications, in Proceedings of the 9th Annual ACM Symposium on Computing in Geometry, 1993, pp. 99-108.

[14] J. Erickson, Dense point sets have sparse Delaunay triangulations, in Proceedings of the 13th ACM-SIAM Symposium on Discrete Algorithms, SIAM, Philadelphia, 2002, pp. 125-134.

[15] T. Erlebach, K. Jansen, And E. Seidel, Polynomial-time approximation schemes for geometric graphs, in Proceedings of the 12th ACM-SIAM Symposium on Discrete Algorithms, 2001, pp. 671-679.

[16] B. Fischl, M. Sereno, And A. Dale, Cortical surface-based analysis II: Inflation, flattening, and a surface-based coordinate system, NeuroImage, 9 (1999), pp. 195-207.

[17] J. Gao, L. J. Guibas, J. Hershberger, L. Zhang, and A. Zhu, Geometric spanners for routing in mobile networks, IEEE J. Selected Areas Commun. Wireless Ad Hoc Networks, 23 (2005), pp. 174-185.

[18] S. Govindarajan, T. Lukovszki, A. Maheshwari, and N. Zeh, I/O efficient well-separated pair decomposition and its applications, in Proceedings of the 8th European Symposium on Algorithms, 2000, pp. 220-231.

[19] J. Gudmundsson, C. Levcopoulos, G. Narasimhan, and M. Smid, Approximate distance oracles for geometric graphs, in Proceedings of the 13th ACM-SIAM Symposium on Discrete Algorithms, SIAM, Philadelphia, 2002, pp. 828-837.

[20] L. Guibas, A. NGuyen, D. Russel, and L. Zhang, Collision detection for deforming necklaces, in Proceedings of the 18th ACM Symposium on Computational Geometry, 2002, pp. 33-42.

[21] W. K. HAle, Frequency assignment: Theory and applications, Proc. IEEE, 68 (1980), pp. 14971513.

[22] H. B. H. III, M. V. Marathe, V. Radhakrishnan, S. S. Ravi, D. J. Rosenkrantz, and R. E. StEARNS, NC-approximation schemes for NP- and PSPACE-hard problems for geometric graphs, J. Algorithms, 26 (1998), pp. 238-274.

[23] D. B. Johnson and D. A. MaLtz, Dynamic source routing in ad hoc wireless networks, in Mobile Computing, T. Imielinski and H. F. Korth, eds., Kluwer Internat. Ser. Engrg. Comput. Sci. 353, Kluwer Academic Publishers, Dordecht, The Netherlands, 1996.

[24] P. KLeIN, Preprocessing an undirected planar network to enable fast approximate distance queries, in Proceedings of the 13th ACM-SIAM Symposium on Discrete Algorithms, SIAM, Philadelphia, 2002, pp. 820-827.

[25] C. Levcopoulos, G. Narasimhan, and M. H. M. Smid, Improved algorithms for constructing fault-tolerant spanners, Algorithmica, 32 (2002), pp. 144-156. 
[26] X.-Y. Li, G. Calinescu, And P.-J. WAN, Distributed construction of a planar spanner and routing for ad hoc wireless networks, in IEEE INFOCOM 2002, New York, June 2002.

[27] C. A. Mead And L. Conway, Introduction to VLSI Systems, Addison-Wesley, Reading, MA, 1980.

[28] G. L. Miller, S.-H. Teng, And S. A. Vavasis, An unified geometric approach to graph separators, in Proceedings of the 32nd Annual IEEE Symposium on Foundations of Computer Science, 1991, pp. 538-547.

[29] G. Narasimhan AND M. Smid, Approximating the stretch factor of Euclidean graphs, SIAM J. Comput., 30 (2000), pp. 978-989.

[30] C. Perkins AND P. BhagWat, Highly dynamic destination-sequenced distance-vector routing $(D S D V)$ for mobile computers, in Proceedings of the ACM SIGCOMM'94 Conference on Communications Architectures, Protocols and Applications, 1994, pp. 234-244.

[31] C. E. Perkins and E. M. Royer, Ad hoc on-demand distance vector routing, in Proceedings of the IEEE Workshop on Mobile Computing Systems and Applications, 1999, pp. 90-100.

[32] W. D. Smith and N. C. Wormald, Geometric separator theorems and applications, in Proceedings of the 39th IEEE Symposium on Foundations of Computer Science, 1998, pp. 232-243.

[33] K. J. Supowit, The relative neighborhood graph with an application to minimum spanning trees, J. ACM, 30 (1983), pp. 428-448.

[34] J. Tenenbaum, V. De Silva, and J. LAngford, A global geometric framework for nonlinear dimensionality reduction, Science, 290 (2000), p. 22.

[35] M. Thorup, Compact oracles for reachability and approximate distances in planar digraphs, in Proceedings of the 42nd IEEE Symposium on Foundations of Computer Science, 2001, pp. 242-251.

[36] M. Thorup And U. Zwick, Approximate distance oracles, in Proceedings of the ACM Symposium on Theory of Computing, 2001, pp. 183-192.

[37] G. Toussaint, The relative neighborhood graph of a finite planar set, Pattern Recognition, 12 (1980), pp. 261-268.

[38] U. ZwICK, Exact and approximate distances in graphs - a survey, in Proceedings of the 9th Annual European Symposium on Algorithms, 2001, pp. 33-48. 